\title{
A Dynamical Systems Construction of a Counterexample to the Finiteness Conjecture
}

\author{
Victor Kozyakin
}

\begin{abstract}
In 1995 J. C. Lagarias and Y. Wang conjectured that the generalized spectral radius of a finite set of matrices can be attained on a finite product of matrices. The first counterexample to this Finiteness Conjecture was given in 2002 by T. Bousch and J. Mairesse. In 2003 V. D. Blondel, J. Theys and A. A. Vladimirov proposed another proof of a counterexample to the Finiteness Conjecture which extensively exploited combinatorial properties of matrix products.

In the paper, it is proposed one more proof of a counterexample of the Finiteness Conjecture fulfilled in a traditional manner of the theory of dynamical systems. It is presented description of the structure of trajectories with the maximal growing rate in terms of extremal norms and associated with them so-called extremal trajectories. The construction of the counterexample is based on a detailed analysis of properties of extremal norms of two-dimensional positive matrices in which the technique of the Gram symbols is essentially used. At last, notions and properties of the rotation number for discontinuous orientation preserving circle maps play significant role in the proof.
\end{abstract}

\section{INTRODUCTION}

Let $\mathbf{A}=\left\{A_{1}, \ldots, A_{r}\right\}$ be a finite set of real $m \times m$ matrices, and $\|\cdot\|$ be a norm in $\mathbb{R}^{m}$. Associate with any finite sequence $\sigma=\left\{\sigma_{1}, \sigma_{2}, \ldots, \sigma_{n}\right\} \in\{1, \ldots, r\}^{n}$ the matrix $A_{\sigma}=A_{\sigma_{n}} \cdots A_{\sigma_{2}} A_{\sigma_{1}}$, and define for any integer $n \geq 1$ two quantities:

$$
\rho_{n}(A)=\max _{\sigma \in\{1, \ldots, r\}^{n}}\left\|A_{\sigma}\right\|^{1 / n}, \quad \bar{\rho}_{n}(A)=\max _{\sigma \in\{1, \ldots, r\}^{n}} \rho\left(A_{\sigma}\right)^{1 / n} .
$$

Then there exists the limit

$$
\rho(A)=\limsup _{n \rightarrow \infty} \rho_{n}(A),
$$

which does not depend on the choice of the norm $\|\cdot\|$. This limit is called the joint spectral radius of the matrix set $\mathbf{A}$. Analogously, there exists the limit

$$
\bar{\rho}(A)=\limsup _{n \rightarrow \infty} \bar{\rho}_{n}(A),
$$

which is called the generalized spectral radius of the matrix set $\mathbf{A}$. As is shown in [2], for finite matrix sets $\mathbf{A}$ the quantities $\rho(\mathbf{A})$ and $\bar{\rho}(\mathbf{A})$ coincide with each other, and for any $n$ the following inequalities hold

$$
\bar{\rho}_{n}(A) \leq \bar{\rho}(A)=\rho(A) \leq \rho_{n}(A) .
$$

In [13] J. C. Lagarias and Y. Wang conjectured that the value $\bar{\rho}(\mathbf{A})$ in fact coincides with $\rho\left(A_{\sigma}\right)^{1 / n}$ for some $n$ and

This work was partially supported by grant $03-01-00258$ of the Russian Foundation for Basic Research and by grant for Scientific Schools 1532.2003.1 of the President of Russian Federation.

V. Kozyakin is with Institute for Information Transmission Problems, Russian Academy of Sciences, Bolshoj Karetny lane 19, Moscow 127994 GSP-4, Russia, kozyakin@iitp.ru $\sigma \in\{1, \ldots, r\}^{n}$. The first counterexample to this conjecture (which got the name Finiteness Conjecture) was proposed in [5], and the corresponding proof was essentially based on the analysis of the so-called Sturmian measures. Later, another proof [3], [4] of the counterexample to the Finiteness Conjecture appeared which extensively exploited combinatorial properties of permutations of products of positive matrices.

In this paper, it is given one more proof of the counterexample to the Finiteness Conjecture fulfilled in a rather traditional manner of the theory of dynamical systems. The proof is based on the technique of the so-called extremal norms (closely related with the usage of functionals Mañé in [5]) and associated with them extremal trajectories for analysis of "the fastest growing trajectories" generated by matrix sets. To our knowledge, it was N. E. Barabanov [1] who first realized the role of extremal norms in the analysis of properties of matrix products. At a later time, the technique of extremal norm was used in different problems related to the investigation of properties of matrix products (see, e.g., [14]). In this paper, we give a more detailed analysis of the properties of extremal norms of two-dimensional positive matrices in which the technique of the Gram symbols, borrowed from [5], is essentially used. At last, in the proposed proof, the notion and properties of the rotation number for discontinuous orientation preserving circle maps [6], [7], [8] play significant role.

\section{EXTREMAL NORMS AND TRAJECTORIES: GENERAL CASE}

One of the key question in the study of properties of matrix sets $\mathbf{A}=\left\{A_{1}, \ldots, A_{r}\right\}$ is how the joint (generalized) spectral radius $\rho(\mathbf{A})$ is related with the rate of growth of solutions of the difference inclusion

$$
x_{n+1} \in\left\{A_{1}, \ldots, A_{r}\right\} x_{n}, \quad n \geq 0,
$$

in which the value of $x_{n+1}$ is chosen from the set of vectors $\left\{A_{1} x_{n}, \ldots, A_{r} x_{n}\right\}$. Notice that each solution of (1) with some choice of the index sequence $\left\{\sigma_{n}\right\}$ satisfies the equation

$$
x_{n+1}=A_{\sigma_{n}} x_{n}, \quad \sigma_{n} \in\{1, \ldots, r\}, \quad n \geq 0 .
$$

Clearly, the converse is also true.

In what follows solutions of (1) will be referred to as trajectories defined by the matrix set $\mathbf{A}$ or simply trajectories of the matrix set $\mathbf{A}$. The set of all trajectories of the matrix set $\mathbf{A}$ will be denoted as $\mathscr{T}(\mathbf{A})$, the set of all trajectories $\left\{x_{n}\right\}_{n=0}^{\infty}$ of the matrix set $\mathbf{A}$ satisfying the initial condition $x_{0}=x$ will be denoted as $\mathscr{T}(\mathbf{A}, x)$. In general, for $r>1$ the map $x \mapsto \mathscr{T}(\mathbf{A}, x)$ is set-valued. In connection with this recall 
some definitions and basic facts of the theory of set-valued maps (see, e.g., [12, §18]).

Let $\mathbb{X}$ and $\mathbb{Y}$ be topological spaces, the map $f: \mathbb{X} \mapsto \mathbb{Y}$ is called upper semi-continuous at a point $x \in \mathbb{X}$ if for any open set $\mathscr{U} \ni f(x)$ there is an open set $\mathscr{V} \ni x$ such that $f(\mathscr{V}) \subseteq \mathscr{U}{ }^{1}$ The map $f$ is called closed (compact) if for any closed (compact) set $\mathscr{G} \subseteq \mathbb{X}$ the set $f(\mathscr{G}) \subseteq \mathbb{Y}$ is also closed (compact). Clearly, each compact map is closed.

Denote the set of all ordered $r$-tuples $\mathbf{A}=\left\{A_{1}, \ldots, A_{r}\right\}$ of real $m \times m$ matrices by $\mathscr{M}_{m, r}$. Then the set $\mathscr{M}_{m, r}$ may be identified in a natural way with $\mathbb{R}^{r m^{2}}$ if to treat entries of the matrices from $\mathbf{A}$ enumerated in some predefined order as coordinates in $\mathbb{R}^{r m^{2}}$. This allows to treat $\mathscr{M}_{m, r}$ as a topological or, when needed, a metric space.

For any matrix set $\mathbf{A}$ the set of trajectories $\mathscr{T}(\mathbf{A})$ is closed in the space $\Omega\left(\mathbb{R}^{m}\right)$, and the map $(\mathbf{A}, x) \mapsto \mathscr{T}(\mathbf{A}, x)$ is compact and upper semi-continuous.

In what follows, our prime point of interest will be socalled irreducible matrix sets. In connection with this recall that the matrix set $\mathbf{A}$ is called irreducible if the matrices from $\mathbf{A}$ have no common invariant spaces except $\{0\}$ and $\mathbb{R}^{m}$. In [9], [10], [11] such a matrix set was called quasicontrollable.

\section{A. Extremal Norms}

In the analysis of the properties of the joint spectral radius ideas introduced by N. E. Barabanov in [1] play an important role. These ideas were further developed in a number of publications amongst which we distinguish [14].

Theorem 1 (N. E. Barabanov): Let the matrix set $\mathbf{A}=$ $\left\{A_{1}, \ldots, A_{r}\right\}$ be irreducible. Then the quantity $\rho$ is the joint (generalized) spectral radius of $\mathbf{A}$ if and only if there exists a norm $\|\cdot\|$ in $\mathbb{R}^{m}$ such that

$$
\rho\|x\|=\max \left\{\left\|A_{1} x\right\|,\left\|A_{2} x\right\|, \ldots,\left\|A_{r} x\right\|\right\} .
$$

A norm satisfying (2) is called an extremal norm for the matrix set A. Remark that for an irreducible matrix set $\mathbf{A}$ any semi-norm $\|\cdot\|$ satisfying (2) is a norm, provided that it does not equal identically to zero. So, in Theorem 1 it is sufficient to suppose that $\|\cdot\|$ is not a norm but only a semi-norm.

The set of extremal norms possesses a variety of strong properties some of which will be described below.

Let $\mathbf{A}$ be an irreducible matrix set, and let $x_{0}$ be a non-zero vector from $\mathbb{R}^{m}$. Denote by $N_{\text {ext }}\left(\mathbf{A}, x_{0}\right)$ the set of all norms $\|\cdot\|$ extremal with respect to the matrix set $\mathbf{A}$, satisfying the calibrating condition $\left\|x_{0}\right\|=1$. Then $N_{\text {ext }}\left(\mathbf{A}, x_{0}\right)$ can be treated as a subset of $C_{\text {loc }}\left(\mathbb{R}^{m}\right) .^{2}$

Theorem 2 (Compactness of the Set of Extremal Norms): Let $x_{0} \neq 0 \in \mathbb{R}^{m}$ and let $\mathbf{A} \in \mathscr{M}_{m, r}$ be an irreducible matrix set. Then there exists a compact neighborhood $\mathscr{A} \subseteq \mathscr{M}_{m, r}$ of $\mathbf{A}$ such that the map $\mathbf{A}^{\prime} \mapsto N_{\text {ext }}\left(\mathbf{A}^{\prime}, x_{0}\right) \subset C_{\text {loc }}\left(\mathbb{R}^{m}\right)$, where $\mathbf{A}^{\prime} \in \mathscr{A}$, is compact and upper semi-continuous.

\footnotetext{
${ }^{1}$ The symbol $f(\mathscr{V})$ is used to denote the set $\cup_{y \in \mathscr{V}} f(y)$.

${ }^{2}$ The symbol $C_{\text {loc }}\left(\mathbb{R}^{m}\right)$ is used to denote the linear topological space of continuous functions defined on $\mathbb{R}^{m}$ with the topology of uniform convergence on bounded subsets from $\mathbb{R}^{m}$.
}

\section{B. Extremal Trajectories and Their Generators}

A trajectory $\left\{x_{n}\right\}$ of the matrix set $\mathbf{A}$ will be called extremal if in some extremal norm $\|\cdot\|$ the following identity holds:

$$
\rho^{-n}(\mathbf{A})\left\|x_{n}\right\| \equiv \text { const. }
$$

From (2) it is seen that for any $x \neq 0$ such an index $\sigma \in$ $\{1,2, \ldots, r\}$ can be found that $\rho^{-1}(\mathbf{A})\left\|A_{\sigma_{n}} x\right\|=\|x\|$. This allows to construct recursively, for any vector $x \neq 0 \in \mathbb{R}^{m}$ and any extremal norm $\|\cdot\|$, an extremal trajectory $\left\{x_{n}\right\}$ satisfying $x_{0}=x$. Hence, for an irreducible matrix set $\mathbf{A}$ the set of extremal trajectories is nonempty.

Clearly, the definition of extremal trajectories depends on the choice of the extremal norm. Nevertheless, as is stated in Lemma 1 below for an irreducible matrix set there are extremal trajectories which are universal in the sense that such trajectories are extremal in each extremal norm.

Lemma 1: For any irreducible matrix set $\mathbf{A} \in \mathscr{M}_{m, r}$ the set of the universal extremal trajectories is not empty.

To prove Lemma 1 it is sufficient to note that any limiting point in the space $\Omega\left(\mathbb{R}^{m}\right)$ of the set of all weighted left-shifts $\left\{\rho(\mathbf{A})^{-k} x_{n+k}\right\}_{n=0}^{\infty}, k \geq 0$, for a given extremal trajectory $\left\{x_{n}\right\}_{n=0}^{\infty}$ will be a universal extremal trajectory of the matrix set A.

Denote by $\mathscr{E}(\mathbf{A}, x)$ the set of all extremal trajectories $\left\{x_{n}\right\}_{n=0}^{\infty}$ of the matrix set $\mathbf{A}$ satisfying the initial condition $x_{0}=x \neq 0$.

Theorem 3: Let $\mathscr{X} \subset \mathbb{R}^{m}$ be a compact set which does not contain the origin and let $\mathbf{A} \in \mathscr{M}_{m, r}$ be an irreducible set of matrices. Then there is a compact neighborhood $\mathscr{A} \subseteq \mathscr{M}_{m, r}$ of $\mathbf{A}$ such that the map $\left(\mathbf{A}^{\prime}, x\right) \mapsto \mathscr{E}\left(\mathbf{A}^{\prime}, x\right)$, where $\mathbf{A}^{\prime} \in \mathscr{A}$, $x \in \mathscr{X}$, is compact and upper semi-continuous.

In order to describe completely an extremal trajectory $\left\{x_{n}\right\}$ one should know not only the information about the sequence $\left\{x_{n}\right\}$ but also the information about the related index sequence $\left\{\sigma_{n}\right\}$. Below, it will be proposed a construction which determines extremal trajectories as all possible trajectories of some set-valued nonlinear dynamical system. Such a construction will allow us to avoid the necessity to describe explicitly the index sequence $\left\{\sigma_{n}\right\}$.

Let $\|\cdot\|_{*}$ be an extremal norm for the matrix set $\mathbf{A}=$ $\left\{A_{1}, \ldots, A_{r}\right\}$. Denote for each $x \in \mathbb{R}^{m}$ by $g(x)$ the set all the vectors $A_{i} x$ such that $\left\|A_{i} x\right\|_{*}=\rho(\mathbf{A})\|x\|_{*}$ for some $i \in$ $\{1, \ldots, r\}$. By the definition of an extremal norm the set $g(x)$ is not empty and consists of no more than $m$ elements. Note also that each map $g(x)$ has a closed graph and satisfies the identity $\|g(x)\|_{*} \equiv \rho(\mathbf{A})\|x\|_{*}$.

Lemma 2: The sequence $\left\{x_{n}\right\}$ is extremal for the matrix set $\mathbf{A}$ in the extremal norm $\|\cdot\|_{*}$ if and only if it satisfies the inclusion $x_{n+1} \in g\left(x_{n}\right)$ for all $n$.

According to Lemma 2 each trajectory of the set-valued map $g(\cdot)$ is extremal in the norm $\|\cdot\|_{*}$. This motivates us to call the map $g(\cdot)$ as the generator of extremal trajectories. In general, the map $g(\cdot)$ can not be described explicitly. Nevertheless, in Section IV for the sets of $2 \times 2$ matrices 
we will be able to obtain a rather detailed description of the properties of the generators of extremal trajectories.

\section{EXTREMAL NORMS: THE CASE OF A PAIR OF TWO-DIMENSIONAL MATRICES}

In this Section, for the case when the set $\mathbf{A}$ consists of two $2 \times 2$ matrices some additional properties of extremal norms and extremal trajectories are established.

\section{A. Definition and Properties of the Matrix Set}

Consider the pair of matrices

$$
A_{0}=\alpha\left\|\begin{array}{ll}
a & b \\
0 & 1
\end{array}\right\|, \quad A_{1}=\beta\left\|\begin{array}{ll}
1 & 0 \\
c & d
\end{array}\right\|,
$$

where $\alpha, \beta>0$ and

$$
b c \geq 1>a, d>0, \quad b c>(1-a)(1-d) .
$$

Associate the ray $t\left(x_{0}, x_{1}\right), t>0$, passing the point $\left(x_{0}, x_{1}\right) \neq 0, x_{0}, x_{1} \geq 0$, with the number $\xi=x_{1} /\left(x_{0}+x_{1}\right) \in$ $[0,1]$. Then the matrix $A_{0}$ maps the ray with the coordinate $\xi$ at the ray with the coordinate $\varphi_{0}(\xi)$, where

$$
\varphi_{0}(\xi)=\frac{\xi}{a(1-\xi)+b \xi+\xi},
$$

while the matrix $A_{1}$ maps the ray with the coordinate $\xi$ at the ray with the coordinate $\varphi_{1}(\xi)$ :

$$
\varphi_{1}(\xi)=\frac{c(1-\xi)+d \xi}{c(1-\xi)+d \xi+1-\xi} .
$$

Under the condition $b c \geq 1$, for any $0 \leq \xi, \zeta \leq 1$ the inequalities $\varphi_{1}(\xi) \geq \varphi_{0}(\zeta)$ hold. Moreover, under conditions (4) both of the functions $\varphi_{0}(\xi)$ and $\varphi_{1}(\xi)$ strictly increase.

Consider also the pair of matrices conjugate to the matrices $A_{0}$ and $A_{1}$ :

$$
A_{0}^{\prime}=\alpha\left\|\begin{array}{ll}
a & 0 \\
b & 1
\end{array}\right\|, \quad A_{1}^{\prime}=\beta\left\|\begin{array}{ll}
1 & c \\
0 & d
\end{array}\right\| .
$$

The matrix $A_{0}^{\prime}$ maps the ray with the coordinate $\xi$ at the ray with the coordinate $\psi_{0}(\xi)$, where

$$
\psi_{0}(\xi)=\frac{b(1-\xi)+\xi}{a(1-\xi)+b(1-\xi)+\xi},
$$

while the matrix $A_{1}^{\prime}$ maps the ray with the coordinate $\xi$ at the ray with the coordinate $\psi_{1}(\xi)$ :

$$
\psi_{1}(\xi)=\frac{d \xi}{1-\xi+c \xi+d \xi} .
$$

\section{B. Structure of the Switching Set}

Denote by $\mathscr{M}^{\sharp} \subset \mathscr{M}_{2,2}$ the set of all matrix sets A consisting of the matrices $A_{0}$ and $A_{1}$ of the form (3) satisfying (4). Clearly, the matrix set $\mathbf{A} \in \mathscr{M}^{\sharp}$ is irreducible. Given some extremal norm $\|\cdot\|$ in $\mathbb{R}^{2}$ corresponding to $\mathbf{A}$, define the sets

$$
X_{i}=\left\{x:\left\|A_{i} x\right\|=\rho\|x\|\right\}, \quad i=0,1 .
$$

Each of these sets is closed, conic (i.e. contains any vector $t x$ along with the vector $x \neq 0$ ), and $X_{0} \cup X_{1}=\mathbb{R}^{2}$ by the definition of an extremal norm. The set $\Theta=X_{0} \cap X_{1}$ will be called the switching set of the extremal norm $\|\cdot\|$. To analyze the structure of the sets $X_{0}, X_{1}$ and $\Theta$ we will need additional data.

Given a pair of vectors $x, y \in \mathbb{R}^{2}$ and a pair of linear functionals $u(w)=\langle u, w\rangle$ and $v(w)=\langle v, w\rangle$, where $u, v, w \in \mathbb{R}^{2}$. Then the Gram symbol of the ordered four-tuple $\{u, v, x, y\}$ is the expression

$$
\begin{aligned}
\left\{\begin{array}{ll}
u & x \\
v & y
\end{array}\right\}:=u(x) v(y)-u(y) & v(x) \equiv \\
& \equiv\langle u, x\rangle\langle v, y\rangle-\langle u, y\rangle\langle v, x\rangle .
\end{aligned}
$$

The geometrical sense of the Gram symbol is that the ordered pair of the vectors $\{x, y\}$ has the same orientation as the ordered pair of the vectors $\{u, v\}$ if and only if the Gram symbol of the corresponding ordered four-tuple of the vectors $\{u, v, x, y\}$ is positive.

Denote by $K_{+}$the cone of vectors in $\mathbb{R}^{2}$ with the nonnegative coordinates.

Lemma 3: Let $x, y \neq 0$ be a pair of the vectors satisfying $x \in X_{0} \cap K_{+}, y \in X_{1} \cap K_{+}$. Then there are such nonzero vectors $u, v \in K_{+}$for which the following relation is valid

$$
\left\{\begin{array}{ll}
A_{0}^{\prime} u & x \\
A_{1}^{\prime} v & y
\end{array}\right\}=\left\langle A_{0}^{\prime} u, x\right\rangle\left\langle A_{1}^{\prime} v, y\right\rangle-\left\langle A_{0}^{\prime} u, y\right\rangle\left\langle A_{1}^{\prime} v, x\right\rangle \geq 0 .
$$

This Lemma is a key point in the analysis of the structure of the sets $X_{0} \cap K_{+}$and $X_{1} \cap K_{+}$.

Theorem 4: Let $\mathbf{A}=\left\{A_{0}, A_{1}\right\}$ be the matrix set defined by (3) and satisfying (4), and let $\|\cdot\|$ be an extremal norm of the matrix set $\mathbf{A}$. Then each of the sets $X_{0} \cap K_{+}$and $X_{1} \cap K_{+}$ is a sector, and the intersection of these sectors is the ray $\Theta=X_{0} \cap X_{1} \cap K_{+}=\left\{t \vartheta: t \in \mathbb{R}_{+}\right\}$passing a nonzero vector $\vartheta \in K_{+},\|\vartheta\|=1$.

Moreover, the vector $\vartheta$ is a single solution to the system of equations $\left\|A_{1} x\right\|=\left\|A_{2} x\right\|$ and $\|x\|=1$, where $x \in K_{+}$, it depends continuously on the matrices $A_{0}, A_{1}$ and the norm $\|\cdot\|$, and belongs to the sector formed in $K_{+}$by the straight lines

$$
\begin{aligned}
& L_{0}=\left\{\left(x_{0}, x_{1}\right): b x_{1}=(1-a) x_{0}\right\}, \\
& L_{1}=\left\{\left(x_{0}, x_{1}\right):(1-d) x_{1}=c x_{0}\right\} .
\end{aligned}
$$

\section{FREQUENCY PROPERTIES OF EXTREMAL TRAJECTORIES: THE CASE OF TWO-DIMENSIONAL MATRICES}

In this Section, the analysis of the properties of the extremal trajectories of the matrix sets $\mathbf{A}=\left\{A_{0}, A_{1}\right\} \in \mathscr{M}^{\sharp}$ will be continued. Our prime goal will be to prove the following statement.

Theorem 5 (on the Switching Frequency): For any extremal trajectory $\left\{x_{n}\right\}$ of the matrix set $\mathbf{A}=\left\{A_{0}, A_{1}\right\} \in \mathscr{M}^{\sharp}$ determined by the equation

$$
x_{n+1}=A_{\sigma_{n}} x_{n}, \quad n=0,1, \ldots,
$$

it is defined the frequency (the switching frequency of the trajectory)

$$
\sigma=\lim _{n \rightarrow \infty} \frac{1}{n} \sum_{i=1}^{n} \sigma_{i}
$$


of applying the matrix $A_{1}$ in the process of computation of the trajectory $\left\{x_{n}\right\}$.

The frequency $\sigma$ does not depend on the choice of the extremal trajectory $\left\{x_{n}\right\}$ or on the index sequence $\left\{\sigma_{n}\right\}$, and so, it may be denoted as $\sigma(\mathbf{A})$. In addition, $\sigma(\mathbf{A})$ depends continuously on matrices of the matrix set $\mathbf{A}$ and takes rational values if and only if the matrix set $\mathbf{A}$ has an extremal trajectory corresponding to a periodic index sequence $\left\{\sigma_{n}\right\}$.

To prove Theorem 5, we will need auxiliary statements and constructions.

\section{A. Generator of Extremal Trajectories}

Fix in $\mathbb{R}^{2}$ a norm $\|\cdot\|$ extremal for the matrix set $\mathbf{A}$, and denote by $X_{0}$ and $X_{1}$ the sets (7) determined by this norm. In this case, the generator of extremal trajectories $g(\cdot)$ (see the definition in Section II-B) will take the form

$$
g(x)=\left\{\begin{array}{cl}
\rho^{-1} A_{0} x, & \text { if } x \in X_{0} \backslash X_{1}, \\
\rho^{-1} A_{1} x, & \text { if } x \in X_{1} \backslash X_{0}, \\
\left\{\rho^{-1} A_{0} x, \rho^{-1} A_{1} x\right\}, & \text { if } x \notin X_{0} \cap X_{0} .
\end{array}\right.
$$

where $\rho=\rho(\mathbf{A})$.

Let us study the structure of the map $g(\cdot)$ in the first quadrant, i.e. in the cone $K_{+}:=\left\{x=\left(x_{1}, x_{2}\right): x_{1}, x_{2} \geq 0\right\}$, in more details. Introduce in $K_{+}$the coordinate system $(\lambda, \xi)$ by setting $\lambda(x)=\|x\|, \xi(x)=x_{2} /\left(x_{1}+x_{2}\right)$ for $x \neq 0 \in K_{+}$. By Theorem 4 in the coordinate system $(\lambda, \xi)$ the map $f$ takes the form of a map with separable variables

$$
f:(\lambda, \xi) \mapsto(\lambda, \Phi)
$$

where

$$
\Phi=\Phi_{\theta}(\xi)=\left\{\begin{array}{cll}
\varphi_{1}(\xi), & \text { if } & \xi \in[0, \theta), \\
\left\{\varphi_{0}(\theta), \varphi_{1}(\theta)\right\} & \text { if } & \xi=\theta, \\
\varphi_{0}(\xi), & \text { if } & \xi \in(\theta, 1] .
\end{array}\right.
$$

Here the functions $\varphi_{0}(\xi)$ and $\varphi_{1}(\xi)$ are defined by (5) and (6). The graphs of the functions $\varphi_{0}(\xi), \varphi_{1}(\xi)$ and $\Phi_{\theta}(\xi)$ are presented in Fig. 1.

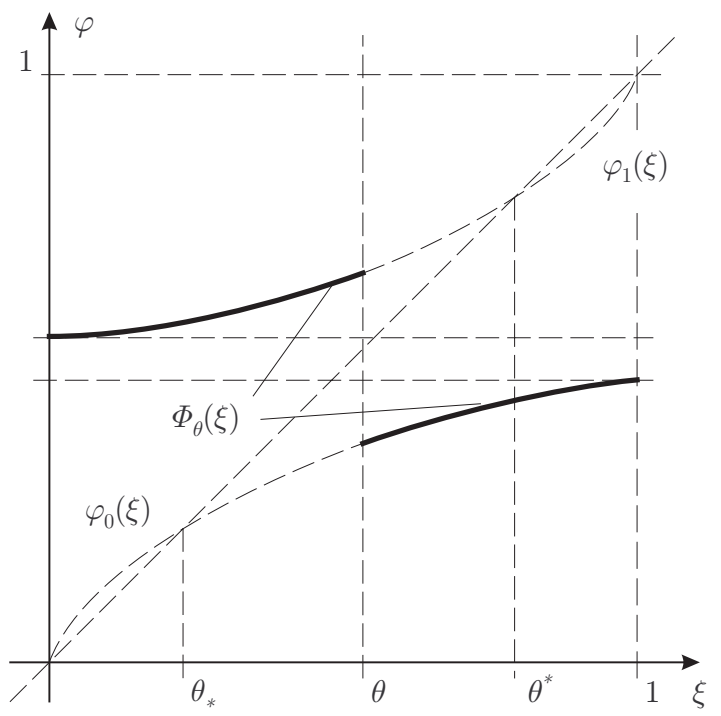

Fig. 1. Graph of the direction function $\Phi_{\theta}(\xi)$
Remark that the coordinate $\lambda(x)$ characterizes "remoteness" of the vector $x$ from the origin of coordinates, while the coordinate $\xi(x)$ characterizes the "direction" of the vector $x$. In accordance with this, $\Phi_{\theta}(\xi)$ can be treated as the direction function of the generator of extremal trajectories.

From Lemma 2, Theorem 4 and the representation (9), (10) of the map $g(\cdot)$ one can get the following description of the extremal trajectories.

Lemma 4: A nonzero trajectory $\left\{x_{n}\right\} \subseteq K_{+}$is extremal for the matrix set $\mathbf{A}=\left\{A_{0}, A_{1}\right\}$ in the extremal norm $\|\cdot\|$ if and only if its elements in the coordinate system $(\lambda, \xi)$ can be represented in the form $x_{n}=\left(\lambda_{n}, \xi_{n}\right)$, where $\lambda_{n} \equiv \lambda_{0}$, and $\left\{\xi_{n}\right\}$ is a trajectory of the set-valued map $\Phi_{\theta}(\cdot)$, i.e.

$$
\xi_{n+1} \in \Phi_{\theta}\left(\xi_{n}\right), \quad n=0,1, \ldots,
$$

whose parameter $\theta$ satisfies the estimates $\theta_{*} \leq \theta \leq \theta^{*}$ with constants $\theta_{*}, \theta^{*} \in(0,1)$ defined by the equalities $\theta_{*}=\varphi_{0}\left(\theta_{*}\right)$ and $\theta^{*}=\varphi_{1}\left(\theta^{*}\right)$.

In addition, the trajectory $\left\{x_{n}\right\}$ satisfies the equation $x_{n+1}=A_{\sigma_{n}} x_{n}$ for $n \geq 0$ with some index sequence $\left\{\sigma_{n}\right\}$ if and only if the trajectory $\left\{\xi_{n}\right\}$ satisfies the equation $\xi_{n+1}=\varphi_{\sigma_{n}}\left(\xi_{n}\right)$ for $n \geq 0$.

Remark that in spite of the fact that the extremal norm $\|\cdot\|_{*}$ is, in general, not known explicitly, the direction function $\Phi_{\theta}(\xi)$ of the generator of extremal trajectories is "defined rather unambiguously" which means that according to (10) it is uniquely defined by the triplet $\left(\varphi_{0}, \varphi_{1}, \theta\right)$ with the only unknown parameter $\theta$. At the same time, by Theorem 4 and relations (8), (10) the parameter $\theta$ is a single-valued function of the matrix set $\mathbf{A}$ and the related extremal norm $\|\cdot\|$, i.e. $\theta=\theta[\mathbf{A},\|\cdot\|]$. From here one can see that the direction function $\Phi_{\theta}(\xi)$ is determined, in the long run, by the matrix set $\mathbf{A}=\left\{A_{0}, A_{1}\right\}$ and by the extremal norm $\|\cdot\|$ corresponding to this set; in the cases when we need stress this dependance it will be used the notation

$$
\Phi_{\theta}(\xi)=\Phi[\mathbf{A},\|\cdot\|](\xi) .
$$

In Lemma 5 below it will be shown that the direction function $\Phi[\mathbf{A},\|\cdot\|]$ depends continuously on the matrix set $\mathbf{A}$ and the extremal norm $\|\cdot\|$. To make said above meaningful, define first the notion of closeness between setvalued functions on the interval $[0,1]$.

Denote by $\mathscr{F}=\mathscr{F}([0,1])$ the set of all set-valued functions $f:[0,1] \mapsto 2^{\mathbb{R}}$ with the closed graphs. In this case the graph $\operatorname{Gr}(f)=\{(x, y): x \in[0,1], y \in f(x)\}$ of the function $f$ is a closed bounded subset of the set $[0,1] \times \mathbb{R} \subset \mathbb{R}^{2}$, and hence, for any pair of functions $f, g \in \mathscr{F}$ it is defined and finite the value

$$
\chi(f, g)=\max \left\{\sup _{x \in \operatorname{Gr}(f)} \bar{\chi}(x, g), \sup _{y \in \operatorname{Gr}(g)} \bar{\chi}(y, f)\right\},
$$

where $\bar{\chi}(u, h)=\inf _{v \in \operatorname{Gr}(h)}|u-v|$ and $|\cdot|$ is some norm in $\mathbb{R}^{2}$. The value $\chi$ is called the Hausdorff distance between the graphs of the maps $f$ and $g$, it is a metric in the space $\mathscr{F}$. In its turn, the space $\mathscr{F}$, being equipped with the metric $\chi$, is complete. 
Lemma 5: Let $x_{0} \in \mathbb{R}^{2}$ be a nonzero vector. Then for any pair $(\mathbf{A},\|\cdot\|)$, where $\mathbf{A} \in \mathscr{M}^{\sharp}$ and $\|\cdot\| \in N_{\text {ext }}\left(\mathbf{A}, x_{0}\right)$, the map $(\mathbf{A},\|\cdot\|) \mapsto \Phi[\mathbf{A},\|\cdot\|]$ is uniquely defined and continuous by the metric of the space $\mathscr{F}$.

Properties of maps, graphs of which are like those presented in Fig. 1, are studied below in more details.

\section{B. Orientation Preserving Discontinuous Circle Maps}

Maps of the interval $[0,1)$ in itself is convenient to treat as maps of the circle $\mathbb{S} \equiv \mathbb{R} / \mathbb{Z}$. Discontinuous maps of the interval $[0,1)$ were studied by various authors (see, e.g., [6], [7], [8] and the bibliography therein), but unfortunately no one of results, known to the author, can be immediately applied to the analysis of the properties of the map $\Phi_{\theta}(\xi)$. For example, in [6] main results are established for the set-valued maps with connected images while in [7], [8] properties of the single-valued discontinuous maps are investigated, whereas in our case $\Phi_{\theta}(\xi)$ is a set-valued map with disconnected images. In view of this, in what follows we will recall basic facts of the theory of orientation preserving discontinuous circle maps, following primarily to the work [6], and then deduce from these results properties of the map $\Phi_{\theta}(\xi)$ needed below.

Let $\eta:[0,1) \rightarrow[0,1)$ be some, in general, discontinuous, set-valued function. The function $h: \mathbb{R} \rightarrow \mathbb{R}$ is called the lift of $\eta$ if it satisfies conditions

$$
\begin{gathered}
h(\xi+1) \equiv h(\xi)+1, \\
\eta(\xi)=h(\xi) \quad(\bmod 1), \quad \xi \in[0,1) .
\end{gathered}
$$

As is easy to see, each circle map has a lift, and conversely, each map $h$ of the straight line in itself satisfying (11) is a lift of the circle map $\eta(\cdot)$ defined by (12).

The map $\eta:[0,1) \rightarrow[0,1)$, treated as a map of the circle $\mathbb{S} \equiv \mathbb{R} / \mathbb{Z}$ in itself, will be called orientation preserving if it has a strictly increasing lift ${ }^{3}$. A strictly increasing lift $h$ of the map $\eta$ will be called standard if it satisfies $h(0)=\eta(0)$. The orientation preserving map $\eta:[0,1) \rightarrow[0,1)$ will be called closed if it has a strictly increasing lift with the closed graph.

Theorem 6: Let $\eta:[0,1) \rightarrow[0,1)$ be an orientation preserving circle map with a closed lift $h$. Let $\left\{\xi_{n}\right\}$ be a trajectory of the map $h$, i.e.

$$
\xi_{n+1} \in h\left(\xi_{n}\right), \quad n=0,1, \ldots .
$$

Then the following assertions are valid:

(i) there is a number $\tau$, not depending on the initial value $\xi_{0}$, such that $\left|\xi_{n} / n-\tau\right| \leq 1 / n$ for $n \geq 1$, and hence $\tau=$ $\lim _{n \rightarrow \infty} \xi_{n} / n$

(ii) if the number $\tau$ is rational of the form $\tau=p / q$ with coprime $p$ and $q$ then the map $\eta(\cdot)$ has a periodic

\footnotetext{
${ }^{3}$ Remark that the lift of a circle map is determined non-uniquely. Nevertheless, just as is in the case of continuous lifts of the circle homeomorphisms, any two strictly increasing lifts of the same circle map (provided that they exist) can differ from each other only on an integer constant [7, Lemma 2]. A detailed description of the structure of singlevalued discontinuous orientation preserving circle maps and their lifts can be found in [7], [8]. The role of the demand of strict increasing of a lift is discussed in Remark 1.
}

point of period $q$, and any trajectory (13) converges to a periodic trajectory of period $q$ as $n \rightarrow \infty$;

(iii) if the number $\tau$ is irrational then all the trajectories (13) have the same limiting set which is either coincide with the whole circle or is the Cantor set;

(iv) the number $\tau$ depends continuously on the graph of the map $h$ in the Hausdorff metric.

According to this Theorem the number $\tau$ is uniquely determined by the map $h$ and does not depend neither on the choice of the initial point $\xi_{0}$ of the trajectory $\left\{\xi_{n}\right\}$ nor on arbitrariness in the construction of the trajectory $\left\{\xi_{n}\right\}$ by (13). So, it is reasonable to denote the number $\tau$ by $\tau(h)$; this number is called the rotation number of the lift $h$. The value $\tau(h)$ is often called also the rotation number of the circle map $\eta$. One should only bear in mind that the rotation number for a circle map is defined modulo integer additives since lifts of the circle map are also defined modulo integer additives.

Remark 1: An orientation preserving circle map was defined above as such a circle map that has a strictly increasing lift. Theorem 6 will be no longer valid if to omit the requirement that the corresponding lift increases strictly.

Remark 2: Theorem 6 was proven in [6] under the additional assumption that the map $\eta$ possesses a lift with the connected graph. As is seen from the formulation of Theorem 6, the requirement of connectedness of the graph is not essential.

One of weak points in the definition of the rotation number $\tau(\eta)$ presented above is that one should perform intermediate steps (such as to construct the lift $h(\cdot)$ and to build the trajectory $\left\{\xi_{n}\right\}$ of the map $\left.h(\cdot)\right)$ to calculate the limit $\tau(\eta)=$ $\lim _{n \rightarrow \infty} \xi_{n} / n$. Therefore, it is desirable to find a method to calculate the rotation number $\tau(\eta)$ directly in terms of the map $\eta$ and its trajectories. To do it, introduce the function

$$
v(\xi)=\left\{\begin{array}{lll}
1 & \text { if } & 0 \leq \xi<\omega \\
0 & \text { if } & \omega \leq \xi<1
\end{array}\right.
$$

where $\omega=\min \{y: y \in \eta(0)\}^{4}$.

Theorem 7: Let $\eta:[0,1) \rightarrow[0,1)$ be an orientation preserving circle map with the closed standard lift $h$. Let $\left\{\zeta_{n}\right\}$ be a trajectory of the map $\eta$, i.e.

$$
\zeta_{n+1} \in \eta\left(\zeta_{n}\right), \quad n=0,1, \ldots .
$$

Then the uniform estimates hold

$$
\left|\frac{1}{n} \sum_{i=1}^{n} v\left(\zeta_{i}\right)-\tau(h)\right| \leq \frac{2}{n}, \quad n=1,2, \ldots,
$$

and so, $\tau(h)=\lim _{n \rightarrow \infty} \frac{1}{n} \sum_{i=1}^{n} v\left(\zeta_{i}\right)$.

\section{Frequency Properties of the Direction Function}

In this Section, we make use of the properties of the circle maps obtained in Section IV-B to analyze the properties of the direction function $\Phi_{\theta}$.

\footnotetext{
${ }^{4}$ Remark that the function $v(\xi)$ is identically equal to zero if $\omega=0$. In this case $h(\xi) \equiv \eta(\xi)$ on the interval $[0,1)$, and so, the function $\eta(\xi)$ strictly increases on $[0,1)$.
} 
Note that the function $\Phi_{\theta}(\xi)$ differs from a function representing an orientation preserving circle map only in that it is defined on the closed interval $[0,1]$ but not on the semiopen one $[0,1)$ as in the case of the circle maps. Let us show that the indicated difference is not essential, and for the function $\Phi_{\theta}(\xi)$ the notion of the rotation number can be defined with all the "good" properties intrinsical to the rotation number of the circle maps.

Theorem 8: Let $\mathbf{A}=\left\{A_{0}, A_{1}\right\} \in \mathscr{M}^{\sharp}$ be the set of matrices (3) satisfying (4), let $\Phi_{\theta}$ be the direction function (10) of some generator of extremal trajectories for the matrix set $\mathbf{A}$ and let $v(\cdot)$ be the function defined by (14). Then for any trajectory $\left\{\xi_{n}\right\}_{n=0}^{\infty}$ of the map $\Phi_{\theta}$ there are valid the nonequalities $\xi_{n} \neq 0,1$, where $n \geq 1$, and there is defined the frequency

$$
\tau=\lim _{n \rightarrow \infty} \frac{1}{n} \sum_{i=1}^{n} v\left(\xi_{i}\right)
$$

with which the elements of the trajectory $\left\{\xi_{n}\right\}$ hit the interval $[0, \omega)$, where $\omega=\varphi_{0}(1)$.

The frequency $\tau$ does not depend neither on the choice of the trajectory $\left\{\xi_{n}\right\}$ nor on the choice of the function $\Phi_{\theta}$. So the frequency $\tau$ may be denoted as $\tau(\mathbf{A})$. In addition, for $\tau(\mathbf{A})$ assertions (i)-(iii) of Theorem 6 are valid, and besides, $\tau(\mathbf{A})$ depends continuously on the matrices of the set $\mathbf{A}$.

Now all is ready to prove Theorem 5. Let $\left\{x_{n}\right\}$ be an extremal trajectory of the matrix set $\mathbf{A}=\left\{A_{0}, A_{1}\right\} \in \mathscr{M}^{\sharp}$ and let $\left\{\sigma_{n}\right\}$ be the corresponding index sequence, i.e.

$$
x_{n+1}=A_{\sigma_{n}} x_{n}, \quad n=0,1, \ldots .
$$

Then by Lemma 4 the numerical sequence $\xi_{n}=\xi\left(x_{n}\right)$, satisfies the relations

$$
\xi_{n+1}=\varphi_{\sigma_{n}}\left(\xi_{n}\right) \in \Phi_{\theta}\left(\xi_{n}\right), \quad n=0,1, \ldots,
$$

with some direction function $\Phi_{\theta}$. At the same time, by Theorem 8 there is defined the frequency

$$
\tau=\lim _{n \rightarrow \infty} \frac{1}{n} \sum_{i=1}^{n} v\left(\xi_{i}\right)
$$

and besides, $\xi_{n} \neq 0,1$ for $n \geq 1$. Therefore, for $n \geq 1$ the value $\xi_{n+1} \in(0,1)$ is obtained from $\xi_{n} \in(0,1)$ by the formula $\xi_{n+1}=\varphi_{0}\left(\xi_{n}\right)$ if and only if $0<\xi_{n+1}<\varphi_{0}(1)$ or, what is the same, if and only if $v\left(\xi_{n+1}\right)=1$. Consequently, $\sigma_{n}=$ $1-v\left(\xi_{n+1}\right)$ for $n \geq 1$ and by Theorem 8 there is the limit

$$
\sigma(\mathbf{A})=\lim _{n \rightarrow \infty} \frac{1}{n} \sum_{i=1}^{n} \sigma_{i}=1-\lim _{n \rightarrow \infty} \frac{1}{n} \sum_{i=1}^{n} v\left(\xi_{i+1}\right)=1-\tau(\mathbf{A}) .
$$

Now, all the assertions of Theorem 5 follow from analogous assertions of Theorem 8 .

\section{Construction of the Counterexample}

At last, start constructing the counterexample to the Finiteness Conjecture.

Lemma 6: Let the matrix set $\mathbf{A}=\left\{A_{0}, A_{1}\right\} \in \mathscr{M}^{\sharp}$ be such that the number $\sigma(\mathbf{A})$ is irrational. Then for any finite sequence of indices $\sigma_{k} \in\{0,1\}, k=1,2, \ldots, n$, the strict inequality $\rho\left(A_{\sigma_{n}} A_{\sigma_{n-1}} \cdots A_{\sigma_{1}}\right)<\rho^{n}(\mathbf{A})$ is valid.
Lemma 6 directly follows from Theorem 5. Due to this Lemma in order to construct the counterexample to the Finiteness Conjecture it is sufficient to prove the existence of at least one of the matrix set $\mathbf{A}=\left\{A_{0}, A_{1}\right\} \in \mathscr{M}^{\sharp}$ for which $\sigma(\mathbf{A})$ is irrational.

Lemma 7: For any set of parameters $a, b, c, d$ satisfying (4) there are positive numbers $\gamma_{*}=\gamma_{*}(a, b, c, d)$ and $\gamma^{*}=$ $\gamma^{*}(a, b, c, d)$ such that for the corresponding matrix set $\mathbf{A}=$ $\left\{A_{0}, A_{1}\right\} \in \mathscr{M}^{\sharp}$ the relations are valid

$$
\sigma(\mathbf{A})=\left\{\begin{array}{lll}
0 & \text { if } & \alpha / \beta>\gamma^{*} \\
1 & \text { if } & \alpha / \beta<\gamma_{*}
\end{array}\right.
$$

Complete the construction of the counterexample. Fix some set of numbers $a, b, c, d$ satisfying (4), and consider the family of the matrix sets $\mathbf{A}$ depending on $\alpha$ and $\beta$ as on parameters. Then by Lemma $7 \sigma(\mathbf{A})=0$ for large values of $\alpha / \beta$ and $\sigma(\mathbf{A})=1$ for small values of $\alpha / \beta$. But by Theorem 5 the value $\sigma(\mathbf{A})$ depends continuously on the matrix set $\mathbf{A}$, and then on $\alpha$ and $\beta$. Hence, $\sigma(\mathbf{A})$ takes all the intermediate values between 0 and 1 when $\alpha$ and $\beta$ vary. In particular, for some $\alpha$ and $\beta$ the number $\sigma(\mathbf{A})$ takes an irrational value. Therefore by Lemma 6 for such $\alpha$ and $\beta$ the generalized spectral radius $\rho(\mathbf{A})$ can not be attained on finite products of matrices from the set $\mathbf{A}$.

\section{REFERENCES}

[1] N. E. Barabanov, "Lyapunov indicator of discrete inclusions. I-III," Autom. Remote Control, vol. 49, no. 2, pp. 152-157, vol. 49, no. 3, pp. 283-287, vol. 49 , no. 5 , pp. $558-565,1988$.

[2] M. A. Berger and Y. Wang, "Bounded semigroups of matrices," Lin. Algebra Appl., vol. 166, pp. 21-27, 1992.

[3] V. D. Blondel, J. Theys, and A. A. Vladimirov, "Switched systems that are periodically stable may be unstable," in Proc. of the Symposium MTNS, Notre-Dame, USA, 2002.

[4] _ - "An elementary counterexample to the finiteness conjecture," SIAM Journal on Matrix Analysis, vol. 24, no. 4, pp. 963-970, 2003.

[5] T. Bousch and J. Mairesse, "Asymptotic height optimization for topical IFS, Tetris heaps, and the finiteness conjecture," J. Amer. Math. Soc., vol. 15 , no. 1 , pp. $77-111,2002$.

[6] R. Brette, "Rotation numbers of discontinuous orientation-preserving circle maps," Set-Valued Analysis, vol. 11, no. 4, pp. 359-371, 2003.

[7] V. S. Kozyakin, "Sturmian sequences generated by order preserving circle maps," Boole Centre for Research in Informatics, University College Cork - National University of Ireland, Cork, Preprint 11/2003, May 2003.

[8] — , "Discontinuous order preserving circle maps versus circle homeomorphisms," Boole Centre for Research in Informatics, University College Cork - National University of Ireland, Cork, Preprint 12/2003, May 2003.

[9] V. S. Kozyakin and A. V. Pokrovskii, "The role of controllability-type properties in the study of the stability of desynchronized dynamical systems," Soviet Phys. Dokl., vol. 37, no. 5, pp. 213-215, 1992.

[10] _ , "Estimates of amplitudes of transient regimes in quasicontrollable discrete systems," Deakin University, Geelong, Australia, CADSEM Report 96-005, 1996.

[11] _ _ "Quasi-controllability and estimation of the amplitudes of transient regimes in discrete systems," Transactions of Russian Academy of Natural Sciences, series MMMIC, vol. 1, no. 3, pp. 128-150, 1997, in Russian.

[12] K. Kuratowski, Topology, Revised and augmented ed. New YorkLondon; Państwowe Wydawnictwo Naukowe, Warsaw: Academic Press, 1966, vol. I.

[13] J. C. Lagarias and Y. Wang, "The finiteness conjecture for the generalized spectral radius of a set of matrices," Lin. Algebra Appl., vol. 214, pp. 17-42, 1995.

[14] F. Wirth, "The generalized spectral radius and extremal norms," Lin. Algebra Appl., vol. 342, pp. 17-40, 2002. 